\title{
Grover Algorithm with zero theoretical failure rate
}

\author{
G. L. Long ${ }^{1,2,3,4}$ \\ ${ }^{1}$ Department of Physics, Tsinghua University, Beijing 100084, P.R. China \\ ${ }^{2}$ Key Laboratory for Quantum Information and Measurements, Ministry of Education, Beijing 100084, P.R. China \\ ${ }^{3}$ Institute of Theoretical Physics, Chinese Academy of Sciences, Beijing, 100080, P. R. China \\ ${ }^{4}$ Center of Atomic, Molecular and Nanosciences, Tsinghua University, Beijing 100084, P. R. China
}

(October 24, 2018)

\begin{abstract}
In standard Grover's algorithm for quantum searching, the probability of finding the marked item is not exactly 1. In this Letter we present a modified version of Grover's algorithm that searches a marked state with full successful rate. The modification is done by replacing the phase inversion by two phase rotation through angle $\phi$. The rotation angle is given analytically to be $\phi=2 \arcsin \left(\frac{\sin \frac{\pi}{(4 J+6)}}{\sin \beta}\right)$, where $\sin \beta=\frac{1}{\sqrt{N}}, N$ the number of items in the database, and $J$ an integer equal to or greater than the integer part of $\left(\frac{\pi}{2}-\beta\right) /(2 \beta)$. Upon measurement at $(J+1)$-th iteration, the marked state is obtained with certainty.
\end{abstract}

PACS numbers: 03.67.Lx, 89.70.+c, 89.80.+h

Grover's quantum search algorithm [i] is an important development in quantum computation. It achieves squareroot speedup over classical algorithms in unsorted database searching. It has extensive applications, because many problems, for instance deciphering the DES encryption scheme, can be reduced to this problem [2]. Starting from an evenly distributed state, Grover algorithm searches the database with

$$
j_{\text {op }}=[(\pi / 2-\beta) /(2 \beta)],
$$

or $j_{o p}+1$ number of times, whichever of $\left(2\left(j_{o p}+1\right)+1\right) \beta$ and $\left(2 j_{o p}+1\right) \beta$ is closest to $\frac{\pi}{2}$. Here $\beta=\arcsin \frac{1}{\sqrt{N}}$ and [] means taking the integer part. $N$ is the number of items in the database. Maximum probability is achieved when measurement is made at the optimal iteration step, and it is

$$
P_{\text {max }}=\sin ^{2}\left(\left(2 j_{o p}+1\right) \beta\right) \cong 1 .
$$

It equals to 1 if $\left(2 j_{o p}+1\right) \beta=\frac{\pi}{2}$. This condition is usually approximately satisfied. This can be seen from table II where values of $j_{o p},\left(2 j_{o p}+1\right) \beta$ for $N$ are given. The deviation $\left(2 j_{o p}+1\right) \beta$ from $\frac{\pi}{2}$ is in the order of $\frac{1}{\sqrt{N}}$. This small deviation becomes negligible when the dimension of the quantum database becomes very large, for instance in deciphering the DES code where $N=2^{56}$ the deviation is only $3 \times 10^{-9}$. Standard Grover algorithm has already achieved high probability, and in most potential applications it is sufficient.

However, in problems where certainty is vital, especially when the dimension is not so big, using a searching algorithm with certainty becomes important. Meanwhile constructing such a quantum search algorithm itself is an interesting issue. In this Letter we present such a modified Grover algorithm.

In fact, two such algorithms already exist. One is given by Brassard et al [3] where the generalized algorithm searches the database $j_{o p}$ iterations with the standard Grover algorithm, and then run one more iteration with a modified algorithm whose step is smaller. Høyer gave another generalization [4], where certainty is achieved by modifying the Grover algorithm and making change to the initial distribution. Our algorithm here complements with these algorithms. In addition, the present algorithm materializes one earlier anticipation [5]. It was pointed out that when the phase inversions are replaced by arbitrary rotations in Grover's algorithm [6], a quantum search algorithm with a smaller iteration can be constructed. Zalka anticipated that this could be used to achive certainty in quantum searching $[5]$ by running a quantum searching algorithm with a smaller step so that at an integer number of iteration, the quantum computer state vector is exactly the marked state. Our algorithm here is just such an algorithm.

The generalized Grover algorithm here starts from the evenly distributed state

$$
\left|\psi_{i}\right\rangle=\frac{1}{\sqrt{N}} \sum_{i}|j\rangle=\frac{1}{\sqrt{N}}(|0\rangle+|1\rangle+\ldots+|\tau\rangle+\ldots+|N-1\rangle)=\sin \beta|1\rangle+\cos \beta|2\rangle,
$$

and the searching operator is

$$
Q=-W I_{0} W I_{\tau}=\left[\begin{array}{cc}
-e^{i \phi}\left(1+\left(e^{i \phi}-1\right) \sin ^{2} \beta\right) & -\left(e^{i \phi}-1\right) \sin \beta \cos \beta \\
-e^{i \phi}\left(e^{i \phi}-1\right) \sin \beta \cos \beta & -e^{i \phi}+\left(e^{i \phi}-1\right) \sin ^{2} \beta
\end{array}\right]
$$


where the matrix expression is written in the following basis

$$
\begin{aligned}
& |1\rangle=|\tau\rangle, \\
& |2\rangle=\frac{1}{\sqrt{N-1}} \sum_{i \neq \tau}|i\rangle,
\end{aligned}
$$

and

$$
\begin{aligned}
& I_{\tau}=I+\left(e^{i \phi}-1\right)|\tau\rangle\langle\tau|, \\
& I_{0}=I+\left(e^{i \phi}-1\right)|0\rangle\langle 0|,
\end{aligned}
$$

where

$$
\begin{aligned}
& \phi=2 \arcsin \left(\frac{\sin \left(\frac{\pi}{4 J+6}\right)}{\sin \beta}\right), \\
& J \geq J_{o p} .
\end{aligned}
$$

Here the two phase rotations are equal which is required by the phase matching condition [7],8]. Certainty in quantum searching is achieved by measuring the quantum computer at $J+1$ iteration. In standard Grover algorithm, $\phi=\pi$. In table III, we gave the angle $\phi$ for some values of $N$. It is seen that in general the phase rotations are very close to $\pi$. We see that at small $N$, the deviation of $\phi$ to $\pi$ is big, and it decreases when $N$ becomes large. The certainty of the algorithm can be examined by direct computation. We will give the detailed derivation of this result in the $S O(3)$ picture of quantum searching algorithm [9].

Equation (7) has a real solutions for $J \geq j_{o p}$, otherwise the solution will be complex. An integer $J \geq j_{o p}$ fixes an phase rotation that searches the marked state with certainty in $J+1$ steps. The lower bound $j_{o p}$ tells us that it can not be faster than the standard Grover algorithm. $J$ can be chosen to be $j_{o p}$, or an integer larger than $j_{o p}$ for convenience.

In the following part, we show the above result and give the expression for the probability during the searching process. We do this in the $S O(3)$ picture introduced recently [9]. In this picture, the quantum search operator (4) corresponds to a rotation in space

$$
R_{Q}=\left[\begin{array}{lll}
R_{11} & R_{12} & R_{13} \\
R_{21} & R_{22} & R_{23} \\
R_{31} & R_{32} & R_{33}
\end{array}\right]
$$

where

$$
\begin{aligned}
& \left.R_{11}=\cos \phi\left(\cos ^{2} 2 \beta \cos \phi+\sin ^{2} 2 \beta\right)+\cos 2 \beta \sin ^{2} \phi\right) \\
& R_{12}=\cos \phi \sin \phi(\cos 2 \beta-1) \\
& R_{13}=-\cos \phi \sin 4 \beta \sin ^{2} \frac{\phi}{2}+\sin 2 \beta \sin ^{2} \phi \\
& R_{21}=-\cos 2 \beta \cos \phi \sin \phi+\left(\cos ^{2} \frac{\phi}{2}-\cos 4 \beta \sin ^{2} \frac{\phi}{2}\right) \sin \phi \\
& R_{22}=\cos ^{\phi}+\cos 2 \beta \sin ^{2} \phi \\
& R_{23}=-\cos \phi \sin 2 \beta \sin ^{2}-\sin 4 \beta \sin ^{2} \frac{\phi}{2} \sin \phi \\
& R_{31}=-\sin 4 \beta \sin 2 \frac{\theta}{2} \\
& R_{32}=\sin 2 \beta \sin \phi, \\
& R_{33}=\cos ^{2} 2 \beta+\cos \phi \sin ^{2} 2 \beta
\end{aligned}
$$

The above rotation is a rotation about the following axis

$$
\vec{l}=\left(\begin{array}{c}
\cos \frac{\phi}{2} \\
\sin \frac{\phi}{2} \\
\cos \frac{\phi}{2} \tan \beta
\end{array}\right),
$$


through an angle $\alpha$

$$
\alpha=4 \arcsin \left(\sin \left(\frac{\phi}{2}\right) \sin \beta\right) .
$$

State vector $|\psi\rangle=(a+b i)|1\rangle+(c+d i)|2\rangle$ is represented by the polarization vector

$$
\vec{r}_{\psi}=\langle\psi|\vec{\sigma}| \psi\rangle=\left(\begin{array}{c}
2(a c+b d) \\
2(-b c+a d) \\
a^{2}+b^{2}-c^{2}-d^{2}
\end{array}\right),
$$

where $\vec{\sigma}=\sigma_{x} \vec{i}+\sigma_{y} \vec{j}+\sigma_{z} \vec{k}$, and $\vec{i}, \vec{j}$ and $\vec{k}$ are the unit vectors along the $x$-, $y$-, and $z$-axis. The initial state $\left|\psi_{i}\right\rangle$ and the marked state $|\tau\rangle$ are represented by

$$
\vec{r}_{i}=\left(\begin{array}{c}
\sin (2 \beta) \\
0 \\
-\cos (2 \beta)
\end{array}\right), \vec{r}_{f}=\left(\begin{array}{l}
0 \\
0 \\
1
\end{array}\right) .
$$

Now we want to find out the angle that we must rotate to shift $\vec{r}_{i}$ to $\vec{r}_{f}$. The equation for a line passing through the origin and parallel to the rotational axis is

$$
\frac{x}{\cos \frac{\phi}{2}}=\frac{y}{\sin \frac{\phi}{2}}=\frac{z}{\cos \frac{\phi}{2} \tan \beta}
$$

and the equation for the plane passing through $(0,0,1)^{T}$ and normal to the rotational axis is

$$
x \cos \frac{\phi}{2}+y \sin \frac{\phi}{2}+(z-1) \cos \frac{\phi}{2} \tan \beta=0 .
$$

The intersecting point of (13) with (14) is

$$
\vec{r}_{o}=\left(\begin{array}{c}
c \cos ^{2}\left(\frac{\phi}{2}\right) \tan \beta \\
c \sin \left(\frac{\phi}{2}\right) \cos \left(\frac{\phi}{2}\right) \tan \beta \\
c \cos ^{2}\left(\frac{\phi}{2}\right) \tan ^{2} \beta
\end{array}\right)
$$

where $c=\frac{1}{1+\cos ^{2} \frac{\phi}{2} \tan ^{2} \beta}$. The angle $\omega$ between $\vec{r}_{i}-\vec{r}_{0}$ and $\vec{r}_{f}-\vec{r}_{0}$ is the angle we have to rotate in a given number of iterations. Using

$$
\left(\vec{r}_{i}-\vec{r}_{o}\right) \cdot\left(\vec{r}_{f}-\vec{r}_{o}\right)=\left|\vec{r}_{i}-\vec{r}_{0}\right|\left|\vec{r}_{f}-\vec{r}_{0}\right| \cos \omega
$$

we find that

$$
\cos \omega=-\cos ^{2} \beta-\cos \phi \sin ^{2} \beta=\cos (2 \arccos x),
$$

where

$$
x=\sin \left(\frac{\phi}{2}\right) \sin \beta
$$

Certainty in finding the marked state is achieved if angle $\omega$ is $J+1$ times of the basic rotation angle $\alpha$ :

$$
\omega=2 \arccos (x)=(J+1) \alpha=4(J+1) \arcsin (x) .
$$

Using the trigonometric relation $\arcsin x+\arccos x=\frac{\pi}{2}$, we obtain

$$
\omega=2\left(\frac{\pi}{2}-\arcsin x\right)=4(J+1) \arcsin (x) .
$$

This gives the result of equation (7). Equation (7) has real solutions for $J \geq j_{o p} . J=j_{o p}$ is the minimum in most cases. In the cases of $N=4$ and $N=1, J=j_{o p}-1$ itself is a solution.

The probability for finding the marked state during the searching can be obtained easily. In the $S O(3)$ picture, the polarization vector at a given iteration is obtained by a simple geometric argument, 


$$
\vec{r}_{j}=\vec{r}_{i} \cos \omega+\vec{l}_{n}\left(\vec{l}_{n} \cdot \vec{r}_{i}\right)(1-\cos \omega)+\left(\vec{l}_{n} \otimes \vec{r}_{i}\right) \sin \omega
$$

where $\vec{l}_{n}$ is the rotational axis (9) normalized to unity. Using the equation (11), the state vector can be determined easily. The probability for finding the marked state is $(z+1) / 2$.

We can also write out the expressions in the $U(2)$ formalism. After diagonalization, the $Q$ operator can be written as,

$$
Q=T \Lambda T^{\dagger}
$$

where

$$
\begin{aligned}
T & =\frac{1}{\sqrt{N_{T}}}\left(\begin{array}{cc}
e^{-i \frac{\phi}{2}}\left(\cos \left(\frac{\phi}{2}\right) \sin \beta+\cos \beta^{\prime}\right) & -\cos \beta \\
\cos \beta & e^{i \frac{\phi}{2}}\left(\cos \left(\frac{\phi}{2}\right) \sin \beta+\cos \beta^{\prime}\right)
\end{array}\right), \\
\Lambda & =\left(\begin{array}{cc}
-e^{i\left(\phi+2 \beta^{\prime}\right)} & 0 \\
0 & -e^{i\left(\phi-2 \beta^{\prime}\right)}
\end{array}\right), \\
\beta^{\prime} & =\alpha / 4=\arcsin \left(\sin \left(\frac{\phi}{2}\right) \sin \beta\right), \\
N_{T} & =\cos ^{2} \beta+\left(\cos \left(\frac{\phi}{2}\right) \sin \beta+\cos \beta^{\prime}\right)^{2} .
\end{aligned}
$$

Successive operations of $Q$ can be written analytically through

$$
Q^{n}=T \Lambda^{n} T^{\dagger}
$$

In summary, a Grover algorithm with certainty is present. Together with the algorithms in Ref. [3] and Ref. [4], there are 3 choices of quantum searching algorithm for finding the marked state with certainty. Our algorithm may be appreciated in cases where the dimension is not big and certainty is important, and in cases where preparation of initial state and the change of the experimental setting during the computation process are difficult.

This work is supported by the Major State Basic Research Developed Program Grant No. G200077400, the China National Natural Science Foundation Grant No. 60073009, the Fok Ying Tung Education Foundation, and the Excellent Young University Teachers' Fund of Education Ministry of China.

[1] L.K. Grover, Phys. Rev. Lett. 79, 325 (1997).

[2] Brassard G., Science 275, 627 - 628 (1997).

[3] Brassard G., Høyer P., Mosca M. and Tapp A., Quantum Amplitude Amplification and Estimation, quant-ph/0005055.

[4] Høyer P., Phys. Rev. A62, 052304 (2001)

[5] Zalka G., quant-ph/9902049

[6] Grover L. K.,Phys. Rev. Lett.,80 4329 (1998)

[7] G.L. Long, Y.S. Li, W.L. Zhang and L. Niu, Phys. Lett. A262, 27 (1999).

[8] Note that the phase condition in Ref. [- is is different from this one, because his initial state is different.

[9] Long G. L. et al, J. Phys. A34, 867 (2001). Also in quant-ph/9911004

TABLE I. Examples of $j_{o p}$ and $\left(2 j_{o p}+1\right) \beta$

\begin{tabular}{ccccccccccc}
\hline \hline$N$ & 2 & 4 & 8 & 100 & 1000 & $10^{4}$ & $10^{6}$ & $10^{8}$ & $10^{10}$ & $2^{56}$ \\
$j_{o p}$ & 0 & 1 & 1 & 7 & 24 & 78 & 784 & 7853 & 78539 & 210828713 \\
$\frac{\left(2 j_{o p}+1\right) \beta}{\frac{\pi}{2}}$ & $\frac{1}{2}$ & 1 & 0.69016 & 0.956528 & 0.986617 & 0.99951 & 0.998857 & 0.999939 & 0.999996 & 0.999999997 \\
\hline \hline
\end{tabular}


TABLE II. Examples of $j_{o p}+1$ and $\phi$

\begin{tabular}{|c|c|c|c|c|c|c|c|c|c|c|}
\hline$N$ & 2 & 4 & 8 & 16 & 100 & 1000 & $10^{4}$ & $10^{6}$ & $10^{8}$ & $10^{10}$ \\
\hline$j_{o p}+1$ & 1 & 1 & 2 & 3 & 8 & 25 & 79 & 785 & 7854 & 78540 \\
\hline$\frac{\phi}{\pi}$ & $\frac{1}{2}$ & 1 & 0.677007 & 0.698709 & 0.748018 & 0.854022 & 0.90089 & 0.989752 & 0.992688 & 0.9973 \\
\hline
\end{tabular}

